EPJ manuscript No.

(will be inserted by the editor)

\title{
Associated strangeness production at threshold
}

P. Kowina ${ }^{1,2}$, M. Wolke ${ }^{1}$, H.-H. Adam ${ }^{3}$, A. Budzanowski ${ }^{5}$, R. Czyżykiewicz ${ }^{4}$, D. Grzonka ${ }^{1}$, M. Janusz ${ }^{4}$, L. Jarczyk ${ }^{4}$, B. Kamys ${ }^{4}$, A. Khoukaz ${ }^{3}$, K. Kilian ${ }^{1}$, T. Lister ${ }^{3}$, P. Moskal ${ }^{1,4}$, W. Oelert ${ }^{1}$, T. Rożek ${ }^{1,2}$, R. Santo ${ }^{3}$, G. Schepers ${ }^{1}$, T. Sefzick ${ }^{1}$, M. Siemaszko ${ }^{2}$, J. Smyrski ${ }^{4}$, S. Steltenkamp ${ }^{3}$, A. Strzałkowski ${ }^{4}$, P. Winter ${ }^{1}$, P. Wüstner ${ }^{6}$, and W. Zipper ${ }^{2}$

${ }^{1}$ IKP, Forschungszentrum Jülich, D-52425 Jülich, Germany

2 Institute of Physics, University of Silesia, PL-40-007 Katowice, Poland

3 IKP, Westfälische Wilhelms-Universität, D-48149 Münster, Germany

' 4 Institute of Physics, Jagellonian University, PL-30-059 Cracow, Poland

${ }^{5}$ Institute of Nuclear Physics, PL-31-342 Cracow, Poland

${ }^{6}$ ZEL, Forschungszentrum Jülich, D-52425 Jülich, Germany

Received: date / Revised version: date

\begin{abstract}
The associated strangeness dissociation at threshold has been studied at the COSY-11 facility measuring the hyperon - and the $K^{+} K^{-}$meson pair production.

Measurements of the near threshold $\Lambda$ and $\Sigma^{0}$ production via the $p p \rightarrow p K^{+} \Lambda / \Sigma^{0}$ reaction [1] at COSY-11 have shown that the $\Lambda / \Sigma^{0}$ cross section ratio exceeds the value at high excess energies (Q $\geq 300 \mathrm{MeV}$ 2] $)$ by an order of magnitude. For a better understanding additional data have been taken between $13 \mathrm{MeV}$ and $60 \mathrm{MeV}$ excess energy.

The near threshold production of the charged kaon-antikaon pair is related to the discussion about the nature of the scalar states in the $1 \mathrm{GeV} / \mathrm{c}^{2}$ mass range, i.e. the $f_{0}(980)$ and $a_{0}(980)$ 3. The interpretation as a $K \bar{K}$ molecule is strongly dependent on the $K-\bar{K}$ interaction which can be studied via the production channel. A first total cross section value on the reaction $p p \rightarrow p p K^{+} K^{-}$at an excess energy of $17 \mathrm{MeV}$ [4] i.e. below the $\phi$ production threshold was measured.
\end{abstract}

PACS. 13.75.-n , 14.20.Jn, 14.40.Aq, 25.40.Ep

\section{THE $\Lambda / \Sigma^{0}$ PRODUCTION RATIO CLOSE TO THRESHOLD}

One of the main investigations of the COSY-11 collaboration is the associated strangeness production of the neutral $\Lambda$ and $\Sigma^{0}$ hyperons in the reactions $p p \rightarrow p K^{+} \Lambda / \Sigma^{0}$. Since the quark structures of these hyperons are analogous to each other one can expect similar production mechanisms. In such a case the cross section ratio $\mathcal{R}_{\Lambda / \Sigma^{0}} \equiv$ $\frac{\sigma\left(p p \rightarrow p K^{+} \Lambda\right)}{\sigma\left(p p \rightarrow p K^{+} \Sigma^{0}\right)}$ should be mainly determined by the isospin relation which leads to $\mathcal{R}_{\Lambda / \Sigma^{0}}=3$ what is in line with the ratio of about 2.5 observed at high excess energies $(\mathrm{Q} \geq 300 \mathrm{MeV})[2$. Very close to threshold, in the range of excess energies $\mathrm{Q} \leq 13 \mathrm{MeV}$, the total cross sections for the $\Lambda$ and $\Sigma^{0}$ hyperon production were measured exclusively at the COSY-11 facility [5] 6] at COSY Jülich [7]. The most remarkable feature of the data [1,8] was that at the same excess energy the total cross section for the $\Sigma^{0}$ production appeared to be about a factor of $28_{-9}^{+6}$ smaller than for the $\Lambda$ particle.

Enhancements in the missing mass distribution at the $\Lambda p$ and $\Sigma N$ thresholds observed in inclusive $K^{+}$production data, taken at SPES 4 [9] in proton-proton scattering at $\mathrm{Q}=252 \mathrm{MeV}$, both having about the same magnitude, suggest a strong $\Sigma N \rightarrow \Lambda p$ final state conversion. This conversion might be responsible for the decrease of the $\Sigma^{0}$ production yield close to threshold as seen in the COSY11 data. Strong $\Sigma N \rightarrow \Lambda p$ conversion effects are also suggested when interpreting the results of $K^{-}$scattering on deuterons [10] where a sharp peak is clearly seen at an effective mass of the $\Lambda$-proton system $\mathrm{m}_{\Lambda p}=2131 \mathrm{MeV} / \mathrm{c}^{2}$ corresponding exactly to the $\Sigma^{0} p$ threshold.

However, in calculations within the Jülich meson exchange model [1] the final state conversion is rather excluded as a dominant origin of the observed $\Sigma^{0}$ suppression. In these calculations both $\pi$ and $K$ exchange are taken into account with inclusion of the final state interaction (FSI) effects. $\Lambda$ production is found to be dominated by kaon exchange, what is in line with the experimental results obtained by the DISTO collaboration [12] at higher excess energies $(\mathrm{Q}=430 \mathrm{MeV})$, where the importance of $K$ exchange is confirmed by a measurement of the polarisation transfer coefficient. On the other hand, in the case of $\Sigma^{0}$ production both $\pi$ and $K$ exchange are found to contribute with about the same strength. A destructive interference of the $\pi$ and $K$ exchange, suggested by Gasparian et al. [11, is able to describe the suppression of the $\Sigma^{0}$ production observed in the close-to-threshold data. 


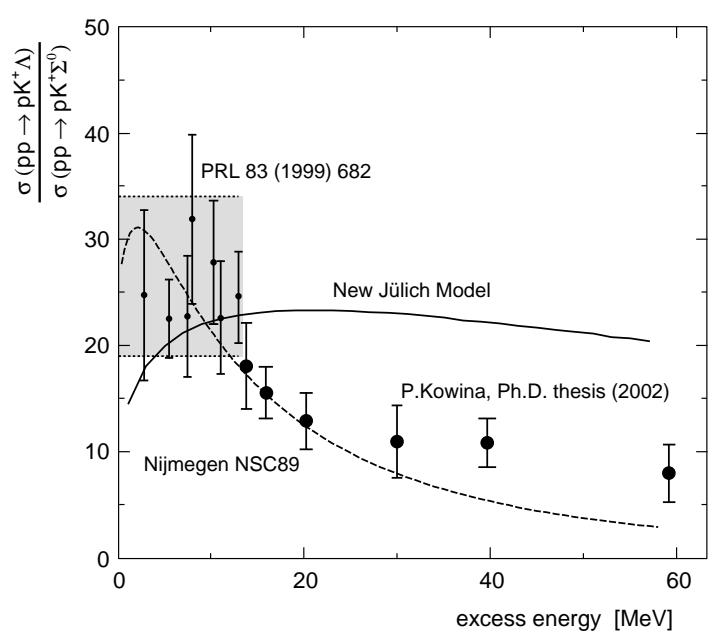

Fig. 1. Energy dependence of the cross section ratio for $\Lambda / \Sigma^{0}$ production in proton-proton collisions. Experimental data within the range up to $13 \mathrm{MeV}$ are from 1, data at higher excess energies from [17. Calculations are performed within the Jülich meson exchange model, assuming a destructive interference of $K$ and $\pi$ exchange [18 and employing the microscopic $Y N$ interaction models Nijmegen NSC89 (dashed line [20]) and the new Jülich model (solid line [22]), respectively.

Studies of the production ratio in [13] consider two different models: either a $\pi$ plus $K$ exchange approach or the excitation of intermediate $N^{*}$ resonances via an exchange of $\pi$ - and heavier non-strange mesons, where the $N^{*}$ 's couple to the $K^{+} Y$ channel [14], however, any interference of the amplitudes are neglected.

The latter mechanism is also taken into account in an effective Lagrangian approach [15] where the strangeness production mechanism is modeled by the exchange of $\pi$, $\rho, \omega$ and $\sigma$ mesons, which excite the nucleon resonances $N^{*}(1650), N^{*}(1710)$, and $N^{*}(1720)$. In both calculations experimental data are reproduced within a factor of two.

The one-boson exchange calculation performed by Laget 16 taking into account interference effects of pion and kaon exchange only by selecting the relative sign for these two mechanism to maximise the cross section reproduce not only the data of the $\Lambda / \Sigma^{0}$ ratio within a factor of two but also the polarisation transfer results of the DISTO experiment [12].

Recent COSY-11 measurements [17] extend the $\Lambda / \Sigma^{0}$ production ratio in proton-proton collisions up to an excess energy of $\mathrm{Q}=60 \mathrm{MeV}$, what allows to study the behaviour of the cross section ratio in the transition region between the low energy range $\mathrm{Q} \leq 13 \mathrm{MeV}$ and data at high excess energies $Q \gg 60 \mathrm{MeV}$. Together with the new [17] and earlier [1] experimental data calculations obtained within the approach of Gasparian et al. 11] are presented in figure 1 where a destructive interference of $\pi$ and $K$ exchange is assumed, with different choices of the hyperon-nucleon interaction model for low-energy scattering in the final state used. The results of the calculations are very sensitive on the off-shell properties of the microscopic hyperon-nucleon interaction.
Both the rather good description of the experimental data very close to threshold by the Jülich model A [19] as well as the fair agreement for the Nijmegen model (dashed line in Fig. (1) with the right tendency of the cross section ratio should not regarded as being very conclusive. In the case of the Nijmegen model an explicit isospin symmetry breaking had to be introduced 20. As a consequence the relation between amplitudes of the $\Sigma^{ \pm} p$ and $\Sigma^{0} p$ channels is not uniquely defined 21.

As already emphasised in [18, both constant elementary amplitudes and only $\mathrm{S}$-waves in the final state may not be justified for excess energies above $20 \mathrm{MeV}$ and thus the calculation based on the new Jülich model 22] (solid line in fig. (1) does not reproduce the excitation function of the experimental cross section ratios.

The data for the $\Lambda$ production in the excess energy range up to $60 \mathrm{MeV}$ are described fairly well by the calculations of the phase space behaviour modified by the $p$ - $\Lambda$ FSI 17] being in line with the scattering parameters from 23. Contrary, in the case of $\Sigma^{0}$ there is almost no deviation from the phase space behaviour in the energy dependence of the cross section for $\Sigma^{0}$ production, which might indicate a very weak $p-\Sigma^{0}$ FSI [17. However, it should be noted that the apparently weak influence of the $p-\Sigma^{0}$ FSI could be feigned by either higher partial wave contributions or an energy dependence of the elementary amplitude 18. Therefore further measurements at an excess energy of $Q \approx 60 \mathrm{MeV}$ are highly desirable to study the angular distribution of the produced $\Lambda$ and $\Sigma^{0}$ hyperons.

\section{EXCLUSIVE KAON-ANTIKAON PRODUCTION AT COSY-11}

Different interpretations of the structure of the scalar resonances $f_{0}(980)$ and $a_{0}(980)$ are known [24,25]. Some motivations for measurements of the $K^{+} K^{-}$production were calculations within the Jülich meson exchange model for the $\pi \pi$ and $\pi \eta$ scattering. The results of these calculation are very sensitive on a strength of the $K \bar{K}$ interaction [3]. Therefore, measurements of the energy dependence of the cross section can help to confirm or exclude the thesis, that the production of $K^{+} K^{-}$leads via excitation of the intermediate resonance. Unfortunately those calculations are done only for $\pi \pi$ scattering, however, similar effects are expected in the case of the $p p$ interaction [26].

From the reconstruction of the full four-momentum vectors for all positively charged ejectiles one obtains the missing mass spectrum of the $\left(p p K^{+}\right)$system shown in the upper part of figure 2 where a clear peak with a resolution (FWHM) of $\approx 2 \mathrm{MeV} / \mathrm{c}^{2}$ is seen at the mass of the charged kaon. The physical background seen is mainly due to the excitation of the hyperon resonances $\Lambda(1405)$ and $\Sigma(1385)$, where the proton originating from the hyperon resonance decay is detected.

Requiring an additional $K^{-}$hit in the dedicated negative particle detector installed at the COSY-11 facility [5] one obtains an almost background free spectrum 


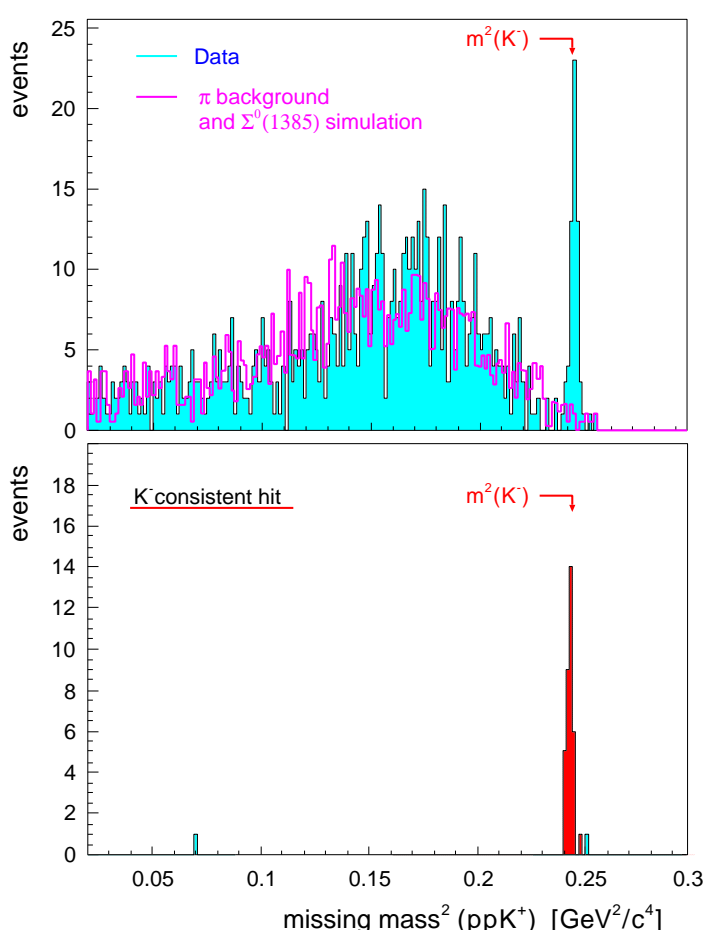

Fig. 2. Missing mass distribution with respect to an identified $\left(\mathrm{ppK}^{+}\right)$subsystem at an excess energy of $17 \mathrm{MeV}$ above the $\mathrm{pp} \rightarrow \mathrm{ppK}^{+} \mathrm{K}^{-}$production threshold without (a) and with (b) $\mathrm{K}^{-}$detection 4 .

of the missing mass of the $p p K^{+}$system shown in the lower part of the Fig. 2] The number of entries in the $K^{-}$peak is slightly reduced compared to the upper figure due to the influence of the kaon decay and acceptance. The analysis resulted in a first total cross section for the elementary $K^{+} K^{-}$production below the $\Phi$ threshold at $Q=17 \mathrm{MeV}$ measured in the proton-proton scattering which is $\sigma=1.80 \pm 0.27_{-0.35}^{+0.28} \mathrm{nb}$ with statistical and systematical errors, respectively [4]. The cross section for the $p p \rightarrow p K^{+} \Lambda$ [1,8 17] reaction which is the elementary $K^{+}$ production is two orders of magnitude higher compared to the cross section for the elementary $K^{-}$production in the $p p \rightarrow p p K^{+} K^{-}$reaction at the corresponding excess energies.

At the present stage it is not possible to judge whether $K^{+} K^{-}$proceeds via a resonant production with the excitation of the $f_{0}(980)$ and $a_{0}(980)$ scalar resonances.

The energy dependence of the total cross section for $K^{+} K^{-}$- below [4] and above 28] the $\Phi$ threshold might be compared to data for $\eta^{\prime}$ 2,27 production, where for an excess energy range $100 \leq \mathrm{Q} \leq 1000 \mathrm{MeV}$ the excitation function is well described by a three-body phase space $\left(\sigma \propto \mathrm{Q}^{2}\right)$. To describe the data below $100 \mathrm{MeV}$ at least the FSI between the final state protons and possibly even the FSI between the final state proton and meson have to be considered.

Not so for the $K^{+} K^{-}$production, where calculations based on a one-boson exchange [29] neglecting FSI effects give significantly different results than simply assuming a four-body phase space behaviour. Contrary to the $\pi N \rightarrow \eta^{\prime} N$ amplitudes the $K^{+} p$ and especially the $K^{-} p$ amplitudes are strongly energy dependent 30. The reason might be a compensation of the interaction of the two strongly interacting subsystems $p p$ and $K^{-} p$ in the final state or an additional degree of freedom given by the fourbody exit channel. In such a case the influence of the FSI effects should be more pronounced at the $K^{+} K^{-}$production threshold [30].

Additional data were taken at the COSY-11 facility at excess energies $10 \mathrm{MeV}$ and $28 \mathrm{MeV}$, i.e. close to the $K^{+} K^{-}$production threshold and slightly below threshold for the $\Phi$ production. The data analysis is presently in progress.

\section{References}

1. S. Sewerin et al., Phys. Rev. Lett. 83 (1999) 682.

2. A. Baldini et al., Total Cross-Sections for Reactions of High-Energy Particles, (Landolt-Börnstein, New Series I/12, Springer, Berlin 1988).

3. O. Krehl, R. Rapp, J. Speth, Phys. Lett. B 390 (1997) 23.

4. C. Quentmeier et al., Phys. Lett. B 515 (2001) 276.

5. S. Brauksiepe et al., Nucl. Instr. \& Meth. A 376 (1996) 397.

6. M. Wolke, Nucl. Phys. News 9 (1999) 27.

7. R. Maier, Nucl. Instr. \& Meth. A 390 (1997) 1

8. J.T. Balewski et al., Phys. Lett. B 420 (1998) 211.

9. R. Siebert et al., Nucl. Phys. A 567 (1994) 819.

10. T.H. Tan, Phys. Rev. Lett. 23 (1969) 395.

11. A. Gasparian et al., Phys. Lett. B 480 (2000) 273.

12. F. Balestra et al., Phys. Rev. Lett. 83 (1999) 1534.

13. A. Sibirtsev et al., e-Print nucl-th/0004022 (2000).

14. K. Tsushima et al., Phys. Rev. C 59 (1999) 369.

15. R. Shyam et al., Phys. Rev. C 63 (2001) 022202.

16. J.-M. Laget, Phys. Lett. B 259 (1991) 24, Nucl. Phys. A 691 (2001) 11c.

17. P. Kowina, Ph.D. thesis, Silesian Univ. Katowice (2002).

18. A. Gasparyan, FZ-Jülich, Matter and Materials 11 (2002) 205.

19. B. Holzenkamp et al., Nucl. Phys. A 500 (1989) 485.

20. P.M.M. Maessen et al., Phys. Rev. C 40 (1989) 2226.

21. J. Haidenbauer, private communications.

22. J. Haidenbauer et al., AIP Conf. Proc. 603 (2001) 421.

23. J.T. Balewski et al., Eur. Phys. J A 2 (1998) 99.

24. U.Thoma, proceedings of this conference.

25. D. Morgan, M.R. Pennington, Phys. Rev. D 48 (1993)

1185; F. Kleefeld et al., Phys. Rev. D 66 (2002) 034007; R.L.

Jaffe, Phys. Rev. D 15 (1977) 267; F.E. Close, Rep. Prog. Phys. 51 (1988) 833; F.E. Close et al., Phys. Lett. B 319 (1993) 291; J. Weinstein, N. Isgur, Phys. Rev. D 41 (1990) 2236; D. Lohse et al., Nucl. Phys. A 516 (1990) 513; Z.S. Wang et al., Nucl. Phys. A 684 (2000) 429c.

26. J. Haidenbauer, FZ-Jülich, Matter and Materials 11 (2002) 225.

27. F. Hibou et al., Phys. Lett. B 438 (1998) 41; P. Moskal et al., Phys. Rev. Lett. 80 (1998) 3202; P. Moskal et al., Phys. Lett. B 474 (2000) 416; F. Balestra et al., Phys. Lett. B 491 (2000) 29.

28. F. Balestra et al., Phys. Rev. C 63 (2001) 024004.

29. A. Sibirtsev et al., Z. Phys. A 358 (1997) 101.

30. A. Sibirtsev, FZ-Jülich, Matter and Materials 11 (2002) 239. 